Draft VERsion April 22, 2018

Preprint typeset using $\mathrm{LAT}_{\mathrm{E}} \mathrm{X}$ style emulateapj v. 5/2/11

\title{
RED SUPERGIANTS AS COSMIC ABUNDANCE PROBES: THE SCULPTOR GALAXY NGC 300
}

\author{
J. Zachary Gazak ${ }^{1}$, Rolf Kudritzki ${ }^{1,2}$, Chris Evans ${ }^{3}$, Lee Patrick ${ }^{4}$, Ben Davies ${ }^{5}$, Maria Bergemann ${ }^{6}$, Bertrand \\ Plez $^{7}$, Fabio Bresolin ${ }^{1}$, Ralf Bender ${ }^{2}$, Michael Wegner ${ }^{2}$, Alceste Z. Bonanos ${ }^{8}$, Stephen J. Williams ${ }^{8}$ \\ Draft version April 22, 2018
}

\section{ABSTRACT}

We present a quantitative spectroscopic study of twenty-seven red supergiants in the Sculptor Galaxy NGC 300. J-band spectra were obtained using KMOS on the VLT and studied with state of the art synthetic spectra including NLTE corrections for the strongest diagnostic lines. We report a central metallicity of $[\mathrm{Z}]=-0.03 \pm 0.05$ with a gradient of $-0.083 \pm 0.014\left[\mathrm{dex} / \mathrm{kpc}^{-1}\right]$, in agreement with previous studies of blue supergiants and H II-region auroral line measurements. This result marks the first application of the J-band spectroscopic method to a population of individual red supergiant stars beyond the Local Group of galaxies and reveals the great potential of this technique.

\section{INTRODUCTION}

The chemical enrichment of galaxies with heavy elements lays a complex roadway across the age of the starforming universe, and techniques to measure metallicity are the astronomer's compass and sextant as the evolution of galaxies is mapped. The primary tools of the trade so far have been the studies of the strongest emission lines of HII regions. They have provided important first hints at the enrichment history of the observable universe which include gradients across the disks of galaxies, a relationship between galactic mass and central metallicity, and metallicity evolution as a function of redshift. Still, these strong-line H II methods are plagued with systematics arising from their empirical calibrations and the complexity of the systems producing the efficiently observable emission lines used (Kewley \& Ellison 2008, Kudritzki et al. 2008, Bresolin et al. 2009). At the same time, it is a basic fact that the stellar population of galaxies provides the drive for chemical enrichment as well as the radiated light used to measure that process. Thus, the natural markers of metallicity are then the stars themselves.

Only recently have developments in the modeling of stellar atmospheres, observational techniques, and statistical methods added the quantitative spectroscopy of supergiant stars to the extragalactic astronomer's toolset. This pioneering work provided a measurement of the radial abundance gradient of NGC 300 using optical spectroscopy of blue supergiant stars and grids of synthetic spectra calculated without assuming local ther-

\footnotetext{
${ }^{a}$ Data collected under ESO Program ID 092.B-0088

1 Institute for Astronomy, University of Hawai'i, 2680 Woodlawn Dr, Honolulu, HI 96822, USA

${ }^{2}$ University Observatory Munich, Scheinerstr. 1, D-81679 Munich, Germany

${ }^{3}$ UK Astronomy Technology Centre, Royal Observatory Edinburgh, Blackford Hill, Edinburgh., EH9 3HJ, UK

${ }^{4}$ Institute for Astronomy, Royal Observatory Edinburgh, Blackford Hill, Edinburgh., EH9 3HJ, UK

${ }^{5}$ Astrophysics Research Institute, Liverpool John Moores University, 146 Brownlow Hill, Liverpool L3 5RF, UK

${ }^{6}$ Institute of Astronomy, University of Cambridge, Madingley Road, Cambridge CB3 0HA, UK

${ }^{7}$ Laboratoire Univers et Particules de Montpellier, Université de Montpellier, CNRS, F-34095 Montpellier, France

8 IAASARS, National Observatory of Athens, GR-15236 Penteli, Greece
}

modynamic equilibrium (LTE). This non-LTE (NLTE) quantitative technique revealed a central metallicity of slightly below solar and a clear gradient across the starforming disk of the galaxy (Kudritzki et al. 2008).

Since that early work, blue supergiants have become a powerful tool for measuring metallicities, gradients, and distances to galaxies in and beyond the Local Group (WLM - Bresolin et al. 2006; Urbaneja et al. 2008; NGC 3109 - Evans et al. 2007; Hosek et al. 2014 IC1613 Bresolin et al. 2007: M33 - U et al. 2009 M81 - Kudritzki et al. 2012; NGC 4258 - Kudritzki et al. 2013. NGC 3621 - Kudritzki et al. 2014). As the technique continues to mature it shows good agreement with the chemical abundances obtained from the nebular emission line method based on the determination of the gas temperature via the weak auroral lines (Bresolin et al. 2009), albeit most recent work (Kudritzki et al. 2013, 2014) indicates a small systematic difference of $\sim 0.1$ dex. By comparison, metallicities derived from any of the host of strong line $\mathrm{H}$ II methods return, at best, consistent trends with huge offsets in overall enrichment level.

A growing body of observational work demonstrates that the quantitative spectroscopy of red supergiant (RSG) stars rivals the precision of metallicity measurements using blue supergiants and is applicable over similar distance scales using existing telescopes and instruments. The pilot near-infrared J-band study of Davies et al. (2010) and high spectral resolution followup by Gazak et al. (2014) -both targeting galactic RSGs-demonstrate the applicability of the technique to stars with roughly solar chemical enrichment. In Davies et al. (2014) and Patrick et al. (2014), the technique is successfully applied to RSGs significantly below solar chemical abundance in the Small and Large Magellanic Clouds and the metal poor Local Group dwarf galaxy NGC 6822.

These studies are aided by continued development of strongly improved quantitative synthetic spectra used to extract stellar parameters. While the underlying grid of MARCS stellar atmospheres is calculated in LTE (Gustafsson et al. 2008), corrections have been developed for NLTE line formation of the strongest diagnostic lines (iron and titanium - Bergemann et al. (2012), silicon Bergemann et al. (2013), and magnesium - Bergemann et al. (2014)). 
The RSG technique will become more important with the next generation telescopes such as the TMT and E-ELT. These telescopes and their instruments will be optimized for observations at infrared wavelengths, using adaptive optics supported multi object spectrographs. In consequence, investigating stars radiating strongly in the near-IR-including red giant, asymptotic giant branch, and red supergiant stars - will have a clear advantage in the future.

In this paper we report another significant step. We have used the multi-IFU NIR spectrograph KMOS at the ESO VLT to test the J-band RSG technique over a wide range of chemical enrichment across the star forming disk of NGC 300 at a distance of $1.88 \mathrm{Mpc}$ (Gieren et al. 2005).

This first application of the technique beyond the Local Group of galaxies is yet another milestone towards a large scale application of the RSG technique to the understanding of the present day chemical enrichment of galaxies undergoing star formation.

After discussing the KMOS observations in $\$ 2$ we outline our analysis procedure in $\$ 3$. For a full description of the $\mathrm{J}$-band technique we direct readers to Gazak et al. (2014). Results and discussion are presented in \$4.

\section{OBSERVATIONS}

\subsection{Target Selection}

We prepared an initial database of RSG candidates using the ACS Nearby Galaxy Survey Treasury (ANGST: Dalcanton et al. 2009), a public database of stellar photometry obtained with Hubble Space Telescope observations. The ANGST catalog contains 6 fields in NGC 300 from the Advanced Camera for Surveys (ACS) and 3 fields observed with the Wide Field and Planetary Camera 2 (WFPC2). The layout of these fields can be seen in Figure 1. We select the red F814W ("I") filter for magnitude cuts. The color cut for both instruments was "V-I", using F555W and F814W filters for ACS, F606W and F814W filters for WFPC2. The cool temperatures and extreme luminosities of RSGs allow for high fidelity selection using such color-magnitude cuts. This is demonstrated in Figure 2 the "RSG plume" (boxed) separates from the population of fainter red objects in $\mathrm{m}_{I}$ magnitude dimension and is distinct from other bright, hotter objects with a red V-I color. Using the ANGST photometry we define RSG candidates as those objects with colors bounded in $\mathrm{V}-\mathrm{I}$ color between 1.2 and 3.5. and having $\mathrm{m}_{I}$ brighter than 19.5, a value selected to achieve our target signal to noise ratio $(\mathrm{SNR})$ of $\sim 100$ (Gazak et al. 2014). Seeking the best spectra possible we divided these targets into two lists separated at $\mathrm{m}_{I}$ $\geq 19.0$ and gave higher priority to the brighter group.

We note that candidate objects within the fainter group could be, in principle, Asymptotic Giant Branch stars (AGB). Stellar evolution models predict some overlap between RGB and so-called super-AGB at $\log \mathrm{L} / \mathrm{L}_{\odot}$ $\sim 4.5$. However, these objects would also be young with ages smaller than 100 Myr with abundances of $\mathrm{Fe}, \mathrm{Si}$, Ti unaltered from their initial composition so that they would also be good tracers of the host galaxys present day abundances.

Because the HST coverage of NGC 300 is incomplete we used overlaps in our database with a more com- plete but shallower catalog of $\mathrm{B}$ and $\mathrm{V}$ photometry (Pietrzyński et al. 2001) to train a $\mathrm{B}-\mathrm{V}$ vs $\mathrm{m}_{V}$ colormagnitude cut. Candidates selected by this method were placed in a third ranked priority group such that HST selected targets would be observed first but that no IFUs would go unused.

As a final adjustment to our RSG candidate list we search for overlaps between our existing candidates and Spitzer photometry of NGC 300 from Khan et al. (2010). The important Spitzer diagnostic for RSG candidacy is the color-magnitude plane defined using IRAC Band 1 $\left(\mathrm{m}_{3.6}\right)$ and the color Band 1 - Band $2([3.6]-[4.5])$. RSGs separate as the brightest $\mathrm{m}_{3.6}$ targets with color blue ward of 0.0 in [3.6]-[4.5] (Verhoelst et al. 2009, Bonanos et al. 2009). We upgrade all objects in our candidate list with overlap as Spitzer RSG candidates to the top priority group. Coordinates and photometric data of our targets finally used for the analysis are provided in Table 5 .

\subsection{Observations}

The resulting candidate catalog was used as input to the KMOS ARM Allocator (KARMA) software for planning the setup of the 24 KMOS Integral Field Units (IFUs) (Wegner \& Muschielok 2008). These $2^{\prime \prime} .8$ square IFUs can be placed over the $7^{\prime} .2 \mathrm{KMOS}$ field of view (shown in Figure 1). We observed an inner and outer field to cover a significant range of the radial extent of NGC300. These fields were observed in 600s integrations over the nights of 2013 October 14, 15, and 16. The 14th and 15th nights were clear and stable at Paranal with median seeing of $\approx 1^{\prime \prime} .1$. Weather degraded slightly on the night of the 16 th with intermittent cloud cover and variable seeing with the median value around $1^{\prime \prime} .3$.

KMOS was operated in nod to sky mode with science integrations of 600 seconds. Telluric standards were observed down each IFU at a frequency of once per every 60 to 90 minutes. KMOS IFU data cubes were flat fielded, wavelength calibrated, and telluric corrected using the KMOS pipeline (v1.3.2) provided publicly as a specific instance of the European Southern Observatory (ESO) Reflex automated data reduction environment (Freudling et al. 2013)

We find inhomogeneities in spectral resolution across the field of view of each KMOS IFU as shown in Figure 3 To correct for this we first measure the effective spectral resolution of night sky lines in each spatial pixel of the inner $12 \times 12$ IFU field. Each sky line is well approximated by a gaussian and for each spatial pixel we calculate the weighted mean of the full width half maxima of those gaussian models. The top panel of Figure 3 shows one such example. The effective resolutions, in this case, vary by up to $\Delta R \sim 300$. We correct this by smoothing the sky and science spectra for each IFU to bring the field to a more constant resolution of $\Delta R \sim 100$ lower than the minimum measured effective resolution. The results of re-measuring effective resolution are displayed in the bottom panel of Figure 3 , which has a global offset in measured resolution but is plotted on the same dynamic scale. The effective resolution after these corrections ranges from the expected value after smoothing to values smaller by $\Delta R \sim 100$.

Once the data cubes are homogenized we extract a science spectrum. We do this by summing over each wave- 


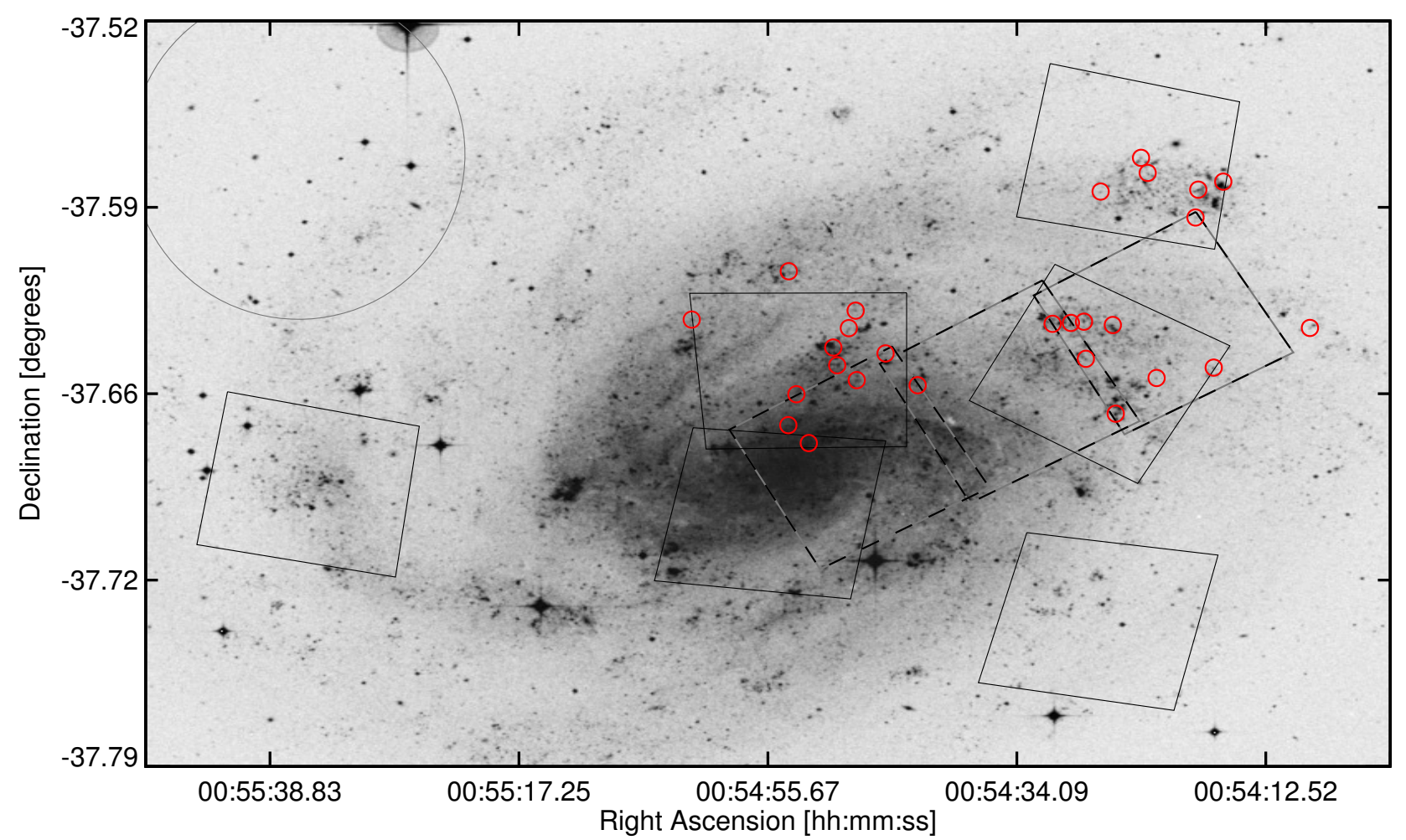

FIG. 1. - The spiral galaxy NGC300. The Hubble ACS fields are overplotted in black, with WFPC2 frames in black dashed with gray. Observed RSGs are circled in red. In the upper left a gray circle denotes the size of the KMOS field of view, within which 24 IFUs can be placed per pointing.
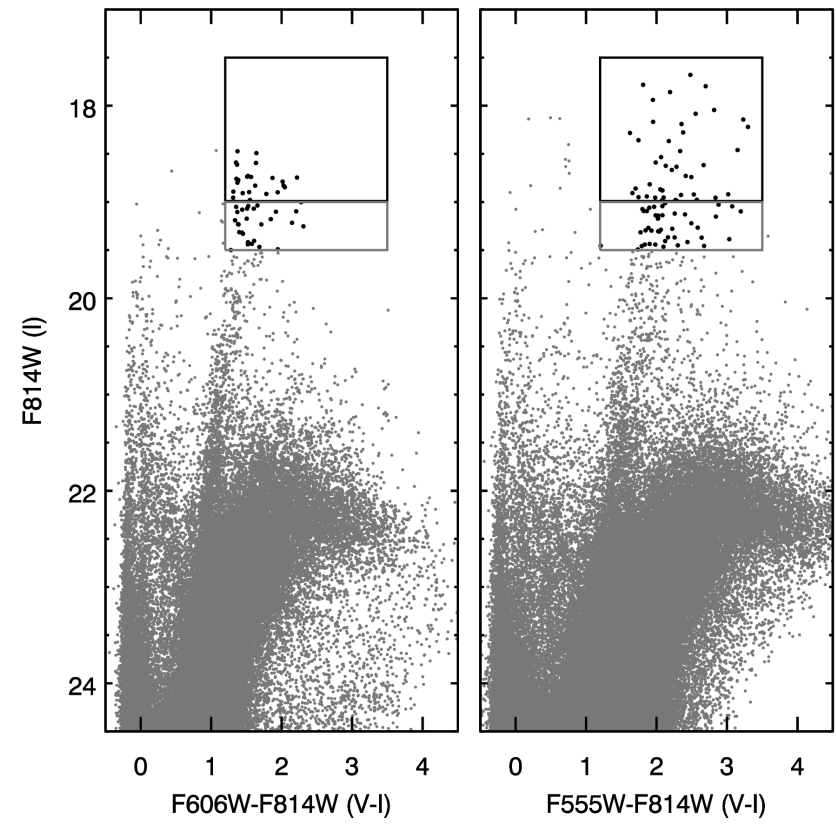

FIG. 2.- Color Magnitude selection for extragalactic RSGs. The left panel shows color vs magnitude for the WFPC observations of NGC 300 while the right panel is the same but for ACS fields. The boxed regions show the brighter "priority one" RSG candidates in this color magnitude space and the fainter "priority two" region below.

length slice in the IFU data cube. In practice, we find that the highest signal to noise is recovered by extracting in a two pixel radius around the peak target flux in the science frame. We suspect that inhomogeneities in wavelength across the field of view play some role in this.

After constructing 1D spectra of object and sky frames, we utilize the ESO tool Skycorr which takes as input those two spectra and scales airglow lines using theoretical knowledge of the night sky lines. The resulting sky spectrum provides a removal of variability in sky emission based on difference in time and sky position of the two observations (Noll et al. 2014).

As each field was observed multiple times, the final science spectra were produced by extracting the median of all individual sky-subtracted spectra. This spectral atlas is plotted in Figure 4

\subsection{Target validation}

In order to verify that our selected targets were indeed members of NGC 300 and not foreground objects, we studied the radial velocities $v_{\text {rad }}$ of each of the stars. We measured $v_{\text {rad }}$ by cross-correlating the reduced spectra with a model spectrum from our grid. The choice of model spectrum was found to be unimportant, producing variations in the measured $v_{\mathrm{rad}}$ of less than $1 \mathrm{~km} \mathrm{~s}^{-1}$, insignificant compared to the other sources of error (see below). The $v_{\text {rad }}$ were then corrected for barycentric motion and converted to the Local Standard of Rest $(L S R)$ using the Starlink package RV.

For the errors on $v_{\text {rad }}$ we first specified a lower limit to the uncertainty of $1 / 10$ th of a pixel, which at the 2 -pixel sampling of KMOS corresponds to $\pm 5 \mathrm{~km} \mathrm{~s}^{-1}$. To this error we added in quadrature the observed spatial variations in wavelength calibration across each IFU, which we found to be $\pm 6 \mathrm{~km} \mathrm{~s}^{-1}$. This gives us an error on each $v_{\text {rad }}$ of $\pm 8 \mathrm{~km} \mathrm{~s}^{-1}$.

To determine what the expected $v_{\text {rad }}$ would be at the position of each target, we used the H I map of West- 

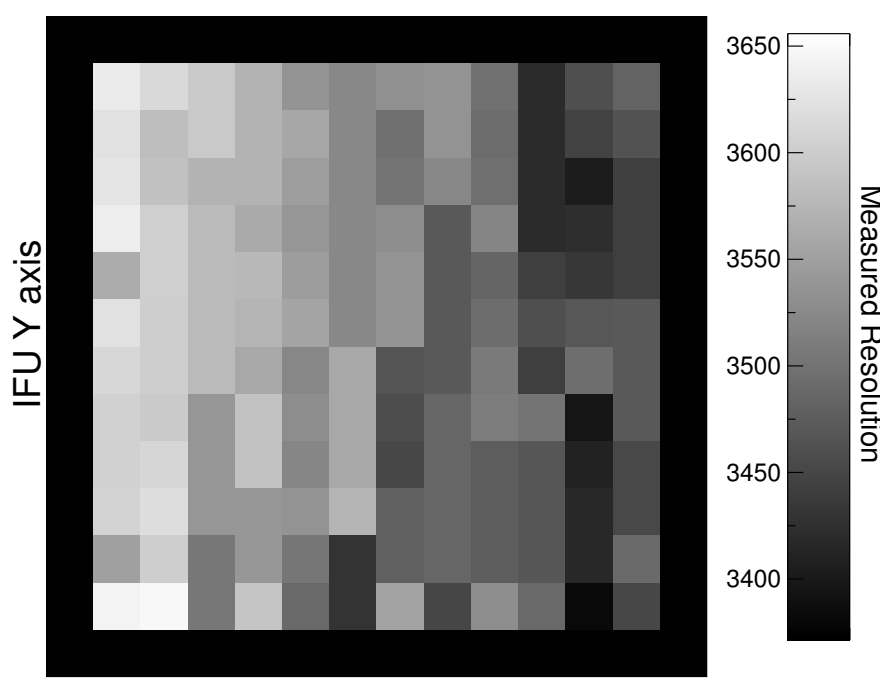

IFU $X$ axis

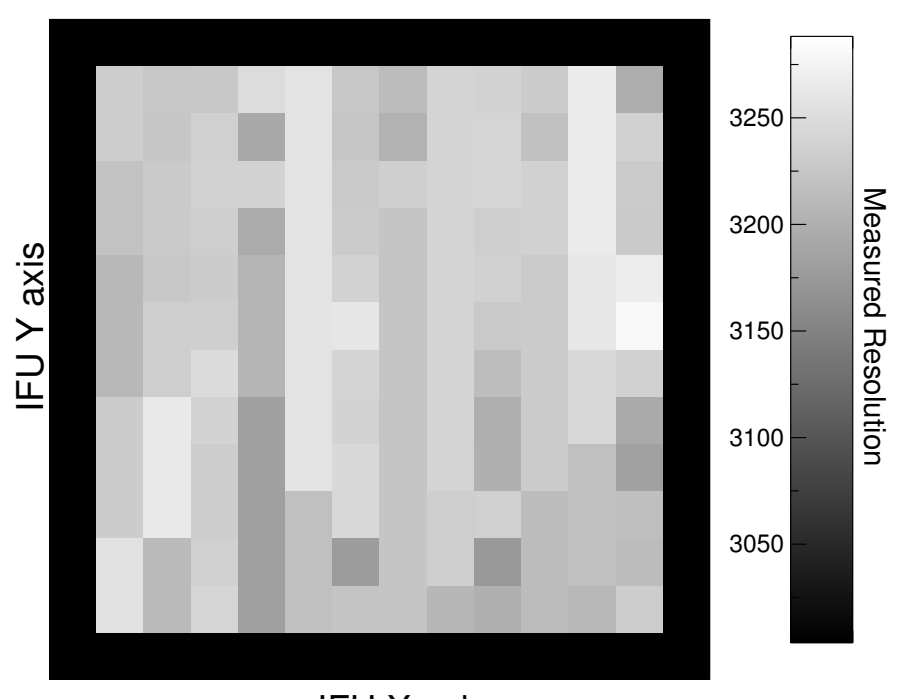

IFU $X$ axis

FIG. 3.- Variation of spectral resolution of the inner $12 \times 12$ spatial pixels of a typical KMOS IFU measured from night sky lines. The top panel shows a typical measurement after a $600 \mathrm{sec}$ exposure. The bottom panel presents the resolution after the homogenizing procedure described in the text is applied.

meier et al. (2011), and their model of NGC 300's rotation curve. These authors fit the observed velocity map with a model which allowed the rotational velocity $v_{\text {rot }}$, inclination $i$ and position angle $P A$ to vary with galactocentric distance. Since the variation in $i$ was found to be small $\left(45 \pm 5^{\circ}\right)$, we kept this parameter fixed at $45^{\circ}$. For $v_{\text {rot }}\left(=40-100 \mathrm{~km} \mathrm{~s}^{-1}\right)$ and $P A\left(=290-330^{\circ}\right)$, we used fits to their observed trends with galactocentric distance. The predicted radial velocity $v_{\text {pred }}$ as a function of position on the plane of the sky relative to the centre of the galaxy $(x, y)$ was then determined from,

$$
v_{\text {pred }}(x, y)=v_{\text {sys }}+v_{\text {rot }} \cos \theta \sin i
$$

where $v_{\text {sys }}=136 \mathrm{~km} \mathrm{~s}^{-1}$ (Westmeier et al. 2011), and $\cos \theta$ is given by,

$$
\cos \theta=\frac{-x \sin (P A)+y \cos (P A)}{r}
$$

and,

$$
r=\left[x^{2}+\left(\frac{y}{\cos i}\right)^{2}\right]
$$

In Fig. 5 we plot the observed versus predicted radial velocities for each star in our sample. The first thing we can say is that there are no obvious candidates for foreground stars in our sample, since such objects would be expected to have low radial velocities of a few $\times 10 \mathrm{~km} \mathrm{~s}^{-1}$. For the majority of our sample, there is excellent agreement between their measured radial velocities and the model rotation curve to within $1 \sigma$. The only deviations greater than $2 \sigma$ are seen at high $v_{\text {rad, }}$, where four datapoints lie $\sim 20 \mathrm{~km} \mathrm{~s}^{-1}$ above the predicted trend. If NGC 300 is similar to the Milky Way in that deviations from the model rotation curve of $20 \mathrm{~km} \mathrm{~s}^{-1}$ are not uncommon (Russeil 2003), then we can say all stars in our sample have radial velocities consistent with being NGC 300 members.

\section{ANALYSIS}

Observed spectra are analyzed through a comparison with synthetic spectra based on a grid of 1D LTE MARCS model atmospheres (Gustafsson et al. 2008). The line formation calculations of the synthetic spectra are carried out in NLTE for the elements iron, titanium, silicon and magnesium, which produce the most critical diagnostic lines prominent in the J-band spectra. The details of the NLTE radiative transfer method and atomic models are described in Bergemann et al. (2012, 2013,2014). All other lines including the (weak) molecular contributions are calculated in LTE. The free parameters of this grid include effective temperature, log gravity, metallicity [Z] (normalized to Solar values, $[\mathrm{Z}]=\log \mathrm{Z} / \mathrm{Z}_{\odot}$ ), and microturbulence $\left(T_{\text {eff }}, \log g,[\mathrm{Z}], \xi\right.$ - see Table 1 for parameter coverage). For the analysis we apply the same technique as described in detail in Gazak et al. (2014). We note, however, that we use an updated set of synthetic spectra calculated with improved oscillator strength for the J-band TiI lines (see Davies et al. 2014).

For a discussion of the limitations and possible improvements of the model atmospheres and the radiative transfer methods used we refer to the publications cited above and the references therein. On the other hand, after the successful tests of the method at Milky Way Gazak et al. 2014) and significantly lower metallicities (Davies et al. 2014, Patrick et al. 2014) we are confident that the method delivers reliable metallicities.

In addition to model parameters we fit for "effective resolution", $R_{\text {eff }}$, an unresolvable combination of the effects of instrument spectral resolution $\mathrm{R}(\delta \lambda / \lambda)$ of each IFU and a very small contribution of macro turbulence in the atmospheres of RSGs. This is done by degrading each model to a set of resolutions ranging from twice the expected spectral resolution downwards until a clear $\chi^{2}$ minimum has been defined. The minimum of a parabolic fit through the $\chi^{2}$ vs. $\mathrm{R}$ is adopted as the best fit $R_{\text {eff }}$ for that combination of model and data. After an initial construction of the $\chi^{2}$ grid allowing each model to be measured with its own ideal $R_{\text {eff }}$ we lock the $R_{\text {eff }}$ to 


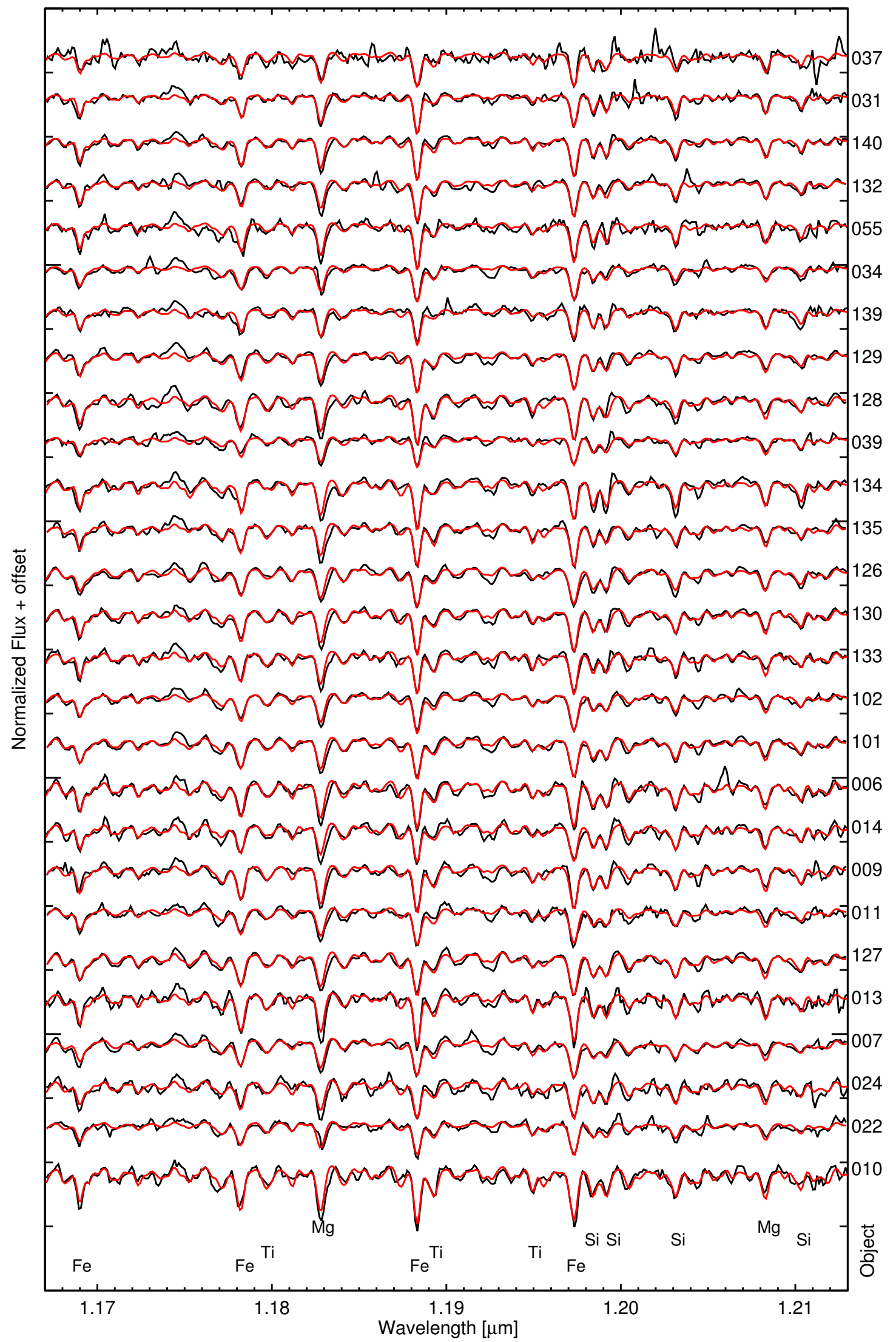

FIG. 4.- NGC 300 RSG spectra plotted in black with corresponding best model fits in red. Diagnostic features are marked. Each object is labeled to the right of the plotted axis and information corresponding to the fit is tabulated by this name in Table 2 


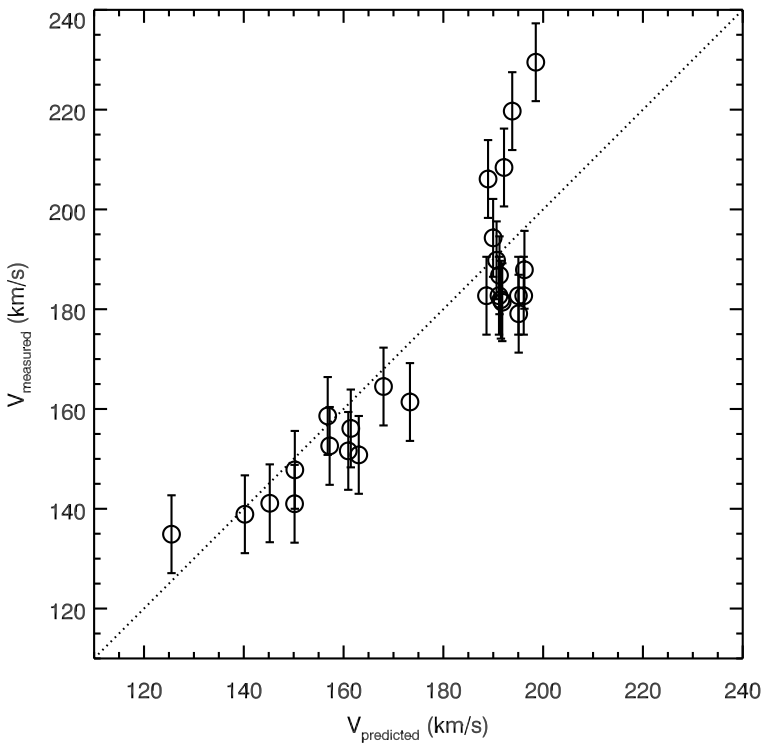

FIG. 5.- The observed radial velocities of our sample stars versus that predicted using the model rotation curve of NGC 300 from Westmeier et al. (2011).

TABLE 1

MARCS MODEL GRID

\begin{tabular}{lcccc}
\hline \hline \multicolumn{1}{c}{ Parameter } & Notation & Min & Max & Spacing \\
\hline Eff. Temperature [K] & $T_{\text {eff }}$ & 3400 & 4000 & 100 \\
& & 4000 & 4400 & 200 \\
Log gravity & $\log g$ & -1.0 & +1.0 & 0.5 \\
Metallicity [dex] & {$[\mathrm{Z}]$} & -1.00 & +1.00 & 0.25 \\
Microturbulence [km/s] & $\xi$ & 1.0 & 6.0 & 1.0 \\
\hline
\end{tabular}

Note. - Parameter grid for MARCS atmospheres (and synthetic spectra) utilized in this work.

the value which matches the minimum of that $\chi^{2}$ and re-calculate. This step is completed for accurate measurement of parameter uncertainties.

Correcting the continuum level of our models to that of the observed spectra starts with selecting the highest model flux point in wavelength bins of characteristic "continuum width" $C W=\lambda / 2 R_{\text {eff }}$ such that the fit samples the full spectrum. We note that at the low resolution of spectra in this work, the true continuum is lost to a blended forest of molecular lines such that we are actually fitting a "pseudocontinuum". While the level of the pseudocontinuum is certainly a function of the physical stellar parameters, especially [Z], we find that no detrimental effects due to this phenomenon appear to resolutions significantly below that of our observed spectra (see Gazak et al. 2014). After removing continuum outliers we fit a smooth third order polynomial through the ratio of observed flux to model flux at the continuum points as a function of wavelength. This function is then applied to scale model to data.

With the continuum normalization procedure described in the preceding paragraph it is important to realize that our spectroscopic J-band method is not affected by the potential presence of circumstellar dust. Though such dust may add a small amount of extinction, it will affect the pseudo continuum and the lines equally and, thus, leave the abundance measurements unaltered.
We measure best fit parameters by isolating the six 2D planes in each parameter pair combination which contain the minimum $\chi^{2}$. As a result, two parameters are locked to the best fit values in each of the six slices. We interpolate the $\chi^{2}$ grid of each plane to a parameter grid with four times the density and take the minimum of that dense grid as the best fit values for the two parameters defining the plane. After completing the procedure for all six planes, the three measurements of each parameter are averaged into a set of best fit parameters (again we refer to Gazak et al. 2014 for all details).

Parameter uncertainties are assessed with a Monte Carlo simulation. This is accomplished by adding 1000 instances of random gaussian noise to a linear interpolation from the model grid to the best fit parameters. The gaussian noise is scaled such that the SNR of this noisy model matches the SNR of the observed spectrum. For each instance we determine fit parameters and for each parameter we define a $1 \sigma$ uncertainty such that the central $68 \%$ of the 1000 measured parameters lies within $\pm 1 \sigma$. The best fit parameters and their derived uncertainties are presented in Table 2

\section{RESULTS AND DISCUSSION}

\subsection{Results}

The parameter fits are in general quite precise with individual metallicities as accurate as 0.10 to 0.15 dex. The exception is object 134 with a high effective temperature just at the edge of our grid of synthetic spectra. It may well be that this object is slightly hotter which would reduce its metallicity and improve the fit of the silicon lines.

In the following, we discuss metallicity and metallicity gradient and the evolutionary status of our objects. The main results are summarized in Figures 6, 7, 8.

\subsection{Metallicity Gradient}

This first measurement of metallicity of the young stellar population of stars across the disk of a spiral galaxy beyond the Local Group using the RSG J-band method requires a careful comparison with previous work.

The most direct comparison of our metallicity results is to the work by Kudritzki et al. (2008), who studied 24 A- and B-type supergiants across the radial extent of this galaxy. They used synthetic NLTE spectra to derive metallicities from an ensemble of heavy atomic species ( $\mathrm{Mg}, \mathrm{Si}, \mathrm{S}, \mathrm{Ti}, \mathrm{Cr}$, and $\mathrm{Fe}$ ) and derived logarithmic metallicities $[\mathrm{Z}]$ relative to the Sun. We display their results in Figure 6. With a linear regression we obtain a central metallicity of $-0.07 \pm 0.09$ dex and a gradient of $-0.081 \pm 0.011$ dex kpc $\mathrm{kp}^{-1}$ from this sample of BSGs. We note that the original Kudritzki et al. (2008) values for central metallicity and gradient were $-0.06 \mathrm{dex}$ and -0.083 dex $\mathrm{kpc}^{-1}$, respectively. We applied the new galactic orientation model of Bresolin et al. (2009) to calculate slightly different galactocentric distances. This resulted in a small change in the central metallicity and metallicity gradient.

Our J-band method is a natural extension of the $\mathrm{Ku}-$ dritzki et al. (2008) technique and the results for our observed RSG sample are also shown in Figure6. We stress that we use an independent but evolutionarily connected population of stars, as blue supergiants below masses of 


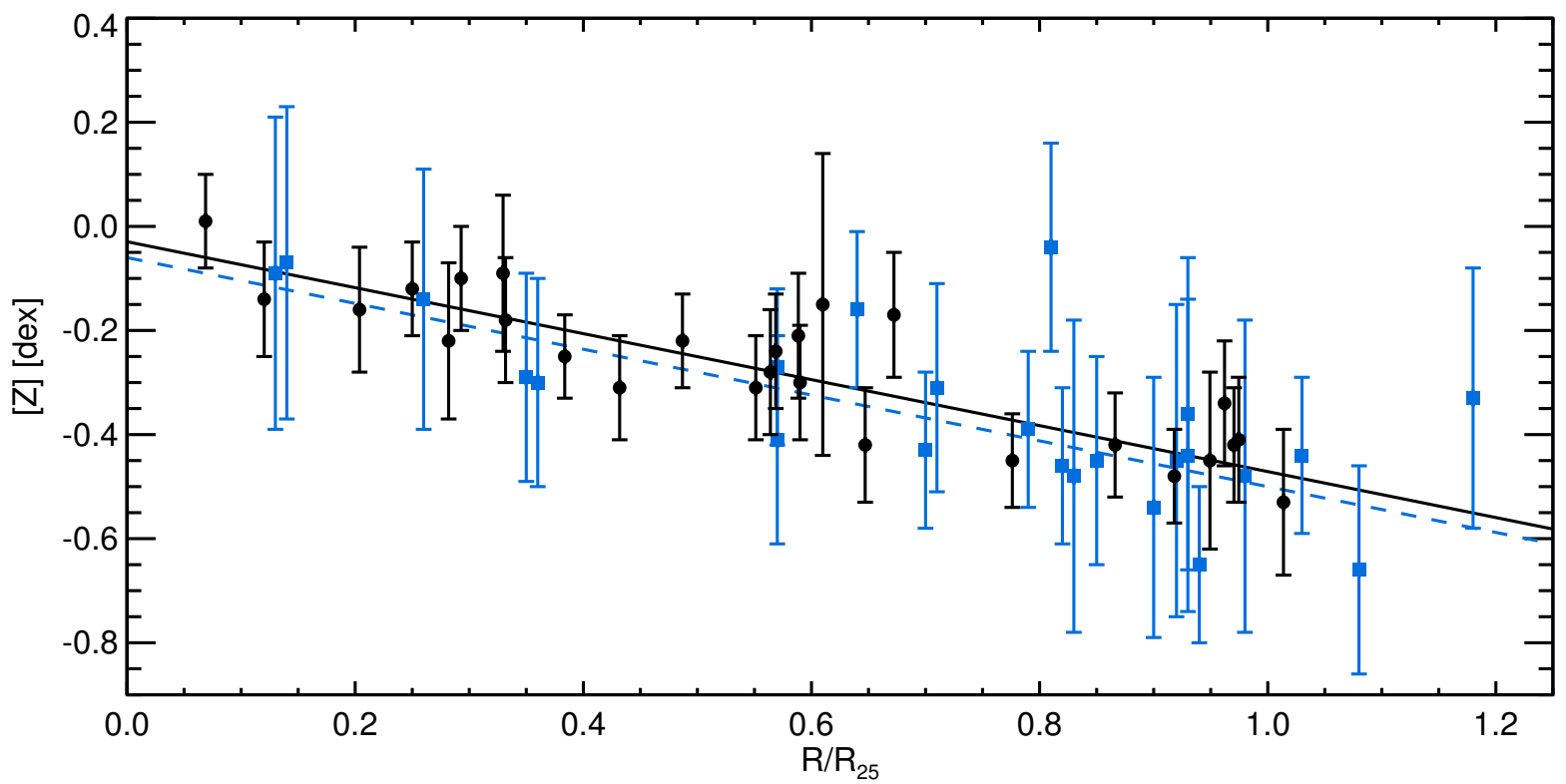

FIG. 6.- The stellar metallicities and metallicity gradients in NGC 300 at 1.88 Mpc. Blue squares are BSGs from Kudritzki et al. (2008) and a linear regression through those points is plotted as a blue dashed line. Black circles and the corresponding solid black linear regression line are RSGs from this work.

TABLE 2

NLTE Stellar Parameters for NGC 300 Red Supergiants

\begin{tabular}{ccccccr}
\hline \hline Target & $\mathrm{R} / \mathrm{R}_{25}$ & $\mathrm{~T}$ eff $[\mathrm{K}]$ & $\log g$ & $\mathrm{Z}[\mathrm{dex}]$ & $\xi[\mathrm{km} / \mathrm{s}]$ & $\mathrm{R}\left[\frac{\lambda}{\delta \lambda}\right]$ \\
\hline 010 & 0.069 & $4096 \pm 40$ & $-0.88 \pm 0.2$ & $+0.01 \pm 0.09$ & $4.4 \pm 0.4$ & 3500 \\
022 & 0.120 & $3535 \pm 70$ & $-0.73 \pm 0.3$ & $-0.14 \pm 0.11$ & $3.1 \pm 0.2$ & 2800 \\
024 & 0.204 & $4153 \pm 40$ & $-0.16 \pm 0.2$ & $-0.16 \pm 0.12$ & $4.0 \pm 0.2$ & 3000 \\
007 & 0.250 & $3912 \pm 70$ & $-0.52 \pm 0.2$ & $-0.12 \pm 0.09$ & $4.0 \pm 0.2$ & 2700 \\
013 & 0.282 & $3875 \pm 50$ & $-0.47 \pm 0.5$ & $-0.22 \pm 0.15$ & $4.3 \pm 0.2$ & 3500 \\
127 & 0.293 & $4220 \pm 30$ & $-0.80 \pm 0.3$ & $-0.10 \pm 0.10$ & $4.3 \pm 0.3$ & 2800 \\
011 & 0.330 & $4063 \pm 70$ & $+0.47 \pm 0.2$ & $-0.09 \pm 0.15$ & $4.1 \pm 0.2$ & 2800 \\
009 & 0.332 & $4075 \pm 40$ & $-0.12 \pm 0.2$ & $-0.18 \pm 0.12$ & $4.3 \pm 0.2$ & 3100 \\
014 & 0.384 & $4260 \pm 30$ & $-0.38 \pm 0.2$ & $-0.25 \pm 0.08$ & $3.6 \pm 0.2$ & 3400 \\
006 & 0.432 & $4205 \pm 90$ & $-0.42 \pm 0.3$ & $-0.31 \pm 0.10$ & $4.1 \pm 0.2$ & 3200 \\
101 & 0.487 & $4228 \pm 42$ & $-0.52 \pm 0.1$ & $-0.22 \pm 0.09$ & $4.2 \pm 0.2$ & 2800 \\
102 & 0.551 & $4010 \pm 147$ & $+0.07 \pm 0.2$ & $-0.31 \pm 0.10$ & $3.9 \pm 0.2$ & 2800 \\
133 & 0.564 & $3953 \pm 52$ & $-0.40 \pm 0.1$ & $-0.28 \pm 0.12$ & $3.6 \pm 0.2$ & 3500 \\
130 & 0.569 & $4123 \pm 30$ & $-0.44 \pm 0.2$ & $-0.24 \pm 0.11$ & $4.1 \pm 0.2$ & 3000 \\
126 & 0.589 & $4251 \pm 60$ & $-0.41 \pm 0.2$ & $-0.21 \pm 0.12$ & $4.1 \pm 0.2$ & 2900 \\
135 & 0.590 & $3953 \pm 53$ & $-0.32 \pm 0.2$ & $-0.30 \pm 0.11$ & $3.8 \pm 0.3$ & 3200 \\
134 & 0.610 & $4400 \pm 20$ & $-0.17 \pm 0.7$ & $-0.15 \pm 0.29$ & $5.0 \pm 0.2$ & 3400 \\
039 & 0.647 & $4240 \pm 50$ & $+0.56 \pm 0.3$ & $-0.42 \pm 0.11$ & $3.0 \pm 0.2$ & 3000 \\
128 & 0.672 & $3776 \pm 63$ & $-0.53 \pm 0.4$ & $-0.17 \pm 0.12$ & $5.0 \pm 0.2$ & 3000 \\
129 & 0.776 & $4113 \pm 50$ & $-0.28 \pm 0.2$ & $-0.45 \pm 0.09$ & $4.1 \pm 0.3$ & 2900 \\
139 & 0.866 & $4280 \pm 30$ & $+0.56 \pm 0.4$ & $-0.42 \pm 0.10$ & $4.1 \pm 0.2$ & 3100 \\
034 & 0.918 & $3833 \pm 44$ & $-0.01 \pm 0.2$ & $-0.48 \pm 0.09$ & $4.1 \pm 0.2$ & 2500 \\
055 & 0.949 & $3981 \pm 119$ & $-0.40 \pm 0.1$ & $-0.45 \pm 0.17$ & $4.1 \pm 0.2$ & 3100 \\
132 & 0.962 & $4160 \pm 43$ & $-0.26 \pm 0.2$ & $-0.34 \pm 0.12$ & $4.5 \pm 0.2$ & 2800 \\
140 & 0.970 & $4141 \pm 30$ & $+0.04 \pm 0.3$ & $-0.42 \pm 0.11$ & $4.1 \pm 0.2$ & 3200 \\
031 & 0.975 & $4270 \pm 40$ & $+0.20 \pm 0.2$ & $-0.41 \pm 0.12$ & $4.0 \pm 0.2$ & 3400 \\
037 & 1.014 & $3912 \pm 50$ & $+0.40 \pm 0.2$ & $-0.53 \pm 0.14$ & $3.1 \pm 0.3$ & 2900 \\
\hline NoTE. $-\mathrm{R}_{25}=5.33 \mathrm{kpc}($ Bresolin et al., 2009) based on a distance of $1.88 \mathrm{Mpc}$ Gieren \\
et al. 2005.
\end{tabular}

$\sim 30 \mathrm{M}_{\odot}$ evolve into the RSGs we observe as they exhaust their core hydrogen. By all means, then, the metallicities obtained with the two techniques should agree. Still, the stellar models and synthetic spectral calculations are independent for our two techniques as the physical conditions of these two populations are distinct. The cool, inflated atmospheres of RSGs contain a host of singly ionized metals and a forest of molecular features which are absent in the hot spectra of BSGs. We also observe our stars in a different wavelength regime subject to separate observational difficulties. Finally, due to the added complexity of RSG atmospheres it is not yet possible to perform calculations in pure NLTE, even though our grid of synthetic spectra do have NLTE calculations for the most critical spectral features. Despite these differences, the J-band technique derives metallicity as a ratio of the solar abundance pattern just as the BSG method does, using strong atomic lines of $\mathrm{Fe}, \mathrm{Ti}, \mathrm{Si}$, and $\mathrm{Mg}$. Our 
observations of NGC300 RSGs then become an excellent test of both methods and of the theoretical calculations from which they draw.

Using our observations and the RSG J-band technique, we measure a central metallicity of $-0.03 \pm 0.05$ dex and a gradient of $-0.083 \pm 0.014 \mathrm{dex} \mathrm{kpc}^{-1}$. This is a stunning agreement between BSGs and the RSG J-band method and certainly represents a breakthrough of the technique.

Bresolin et al. (2009) provide an important second measurement of the central metallicity and gradient of NGC 300 using measurements of H II region auroral lines. Studies using this "direct method" of measurement agree with abundances derived from populations of stars in environments where metallicity is below solar, for example, dwarf galaxies (Stasińska 2005, Bresolin et al. 2006; Lee et al. 2006), and for some regions in the Milky Way and M33 (MW: Rolleston et al. 2000, Deharveng et al. 2000, Daflon \& Cunha 2004, M33: Vilchez et al. 1988; Urbaneja et al. 2005; Bresolin 2011). In this technique the [O III auroral lines give access to a key physical parameter, the electron temperature $T_{e}$, which can disentangle the effect of line strengths based on oxygen abundance and temperature. Bresolin et al. (2009) find a central oxygen abundance of $12+\log (\mathrm{O} / \mathrm{H})=8.57 \pm 0.02$ and a gradient of $-0.077 \pm 0.006 \mathrm{dex} \mathrm{kpc}^{-1}$. The gradient is slightly shallower than for the RSGs and BSGs, but the difference is small. The comparison of central metallicity depends on the assumed value for the solar oxygen abundance. Choosing $12+\log (\mathrm{O} / \mathrm{H})_{\odot}=8.69$ Asplund et al. 2009) returns a $\mathrm{H}$ II region central metallicity value of $\mathrm{Z}=-0.12 \pm 0.02$. While this value agrees with the BSG result it indicates a small offset $(\sim 0.09 \mathrm{dex})$ relative to the RSGs. We note, however, that such an offset is also found in the recent work by Kudritzki et al. (2013. 2014 ) in the spiral galaxies NGC 4258 and 3621 when $\mathrm{BSG}$ and $T_{e} \mathrm{H}$ II region metallicities are compared. A possible reason for this discrepancy is the depletion of nebular oxygen on dust grains as, for instance, suggested by Zurita \& Bresolin (2012). On the other hand, we note that comparing the metallicities obtained with the three methods at $\mathrm{R} / \mathrm{R}_{25}=1$ the situation looks much better. We find $[\mathrm{Z}]=-0.50,-0.47,-0.53$ for the BSG, RSG, and $\mathrm{HII}$ auroral line methods, respectively. Given that the three methods are entirely different the agreement is striking. The measurements from this work are tabulated with the discussed work from the literature in Table 3.

\subsection{Stellar Evolution}

Stellar evolution theory predicts a shift of the Hayashi limit (the minimum temperature of an RSG evolutionary track, when stars are nearly fully convective) towards higher effective temperature with decreasing metallicity. For a stellar evolutionary track with solar metallicity and a mass of $\mathrm{M}=15 \mathrm{M}_{\odot}$ a minimum temperature of $\sim 3600 \mathrm{~K}$ is obtained (Ekström et al. 2012, Meynet \& Maeder 2002), whereas models with SMC like metallicity (Georgy et al. 2013) have a minimum temperature of $\sim 4100 \mathrm{~K}$. In contrast to this prediction, the RSG J-band spectroscopy in the Milky Way, LMC \& SMC, and NGC 6822 Gazak et al. 2014; Davies et al. 2014; Patrick et al. 2014) does not show such a trend and leads to the conclusion that the temperatures of RSGs are independent of metallicity. We can use our observations in NGC 300 as an additional test in this regard since we encounter a range of metallicities between solar in the center of the NGC 300 disk down to $1 / 3$ solar at the isophotal radius. Accordingly, Figure 7 shows a plot of effective temperature against metallicity. It confirms the absence of a trend at least in the metallicity range covered. In 1D evolutionary models, the temperature of the Hayashi limit (i.e. the maximum size of a star) is governed by the treatment of convection, in particular by the mixinglength parameter $\alpha$. The value of $\alpha$ is typically tuned in order to reproduce the properties of the Sun, but then is fixed at this value for stars of all masses, ages and metallicities. This fixed value of $\alpha$ typically results in evolutionary paths for RSGs which are 'inclined', decreasing in $T_{\text {eff }}$ as $L$ increases, as well as producing trends of lower $T_{\text {eff }}$ for RSGs with higher initial masses, and higher average $T_{\text {eff }}$ at lower metallicities (Meynet \& Maeder 2000, Eldridge et al. 2008; Brott et al. 2011).

Recently, studies using 3-D hydrodynamical simulations of convection have attempted to empirically model the evolution of $\alpha$ for stars across a range of $T_{\text {eff }}, \log g$ and metallicity (Trampedach et al. 2014, Magic et al. 2015). The nature of the simulations used in these studies meant that it was not possible to cover the parameter space occupied by RSGs, and instead focussed on stars of higher gravities and temperatures. However, extrapolating from the observed trends in Magic et al. (2015), the results seem to suggest that $\alpha$ should increase at lower metallicity for a fixed $T_{\text {eff }}$ and $\log g$. If correct, this would cause RSGs at a fixed initial mass and evolutionary stage to become hotter at lower metallicity, exacerbating the trend seen in evolutionary models rather than counteracting it. In effect this is the opposite to what we find in our results, that the average effective temperature of RSGs seems to be independent of metallicity.

The discussion of Figure 7 is, of course, based on the assumption that our determination of effective temperatures using the $\mathrm{J}$-band technique is not affected by spurious systematic effects which lead to erroneous effective temperatures which are always very similar even for significantly different metallicities. A first clear indication that this is not the case comes from the work by Davies et al. (2013). This comprehensive study of RSGs in the LMC and SMC determined effective temperatures using ESO VLT XShooter observations of SEDs covering the full spectral range from the near-UV to the nearIR. It was found that the RSG effective temperatures in both galaxies are very similar and do not seem to be affected by the difference in metallicity. Moreover, Davies et al. (2014) recently re-investigated the same sample of RSGs applying the spectroscopic J-band technique and found effective temperatures consistent with the fits of full SEDs indicating that the J-band method provides robust estimates of effective temperatures. For a more detailed discussion of RSG temperature determinations we refer to Davies et al. (2013) and Gazak et al. (2014).

We construct a Hertzsprung-Russell diagram (HRD) to compare our results with stellar evolution theory. We calculate bolometric luminosities for program stars using F814W (I) band photometry from Hubble and the bolometric correction recipes of Davies et al. (2013). We adopt the distance modulus of $\mu=26.37 \pm 0.05$ from the work by Kudritzki et al. (2008) and Gieren et al. (2005). These luminosities are plotted against the effective temperatures from our spectral fit in the HRD of Figure 8. 


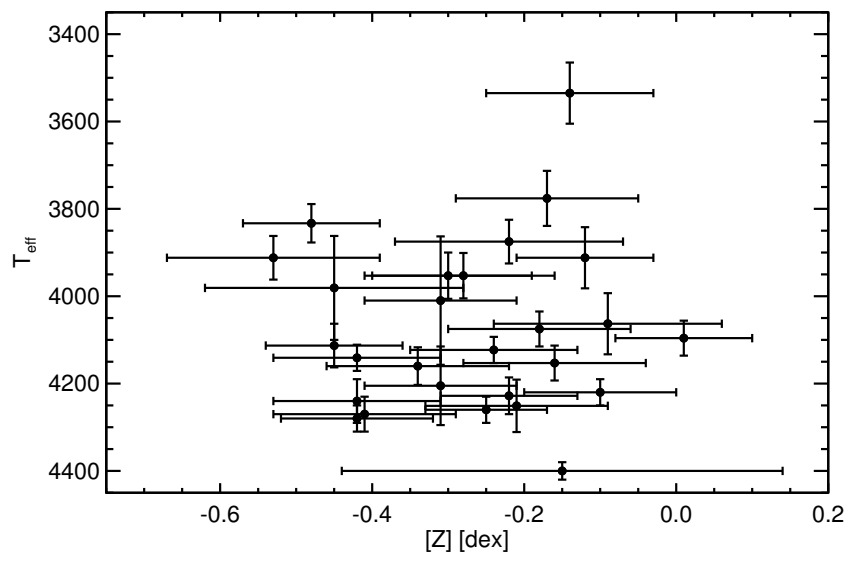

FIG. 7.- Effective temperature of our RSG targets as a function of metallicity.

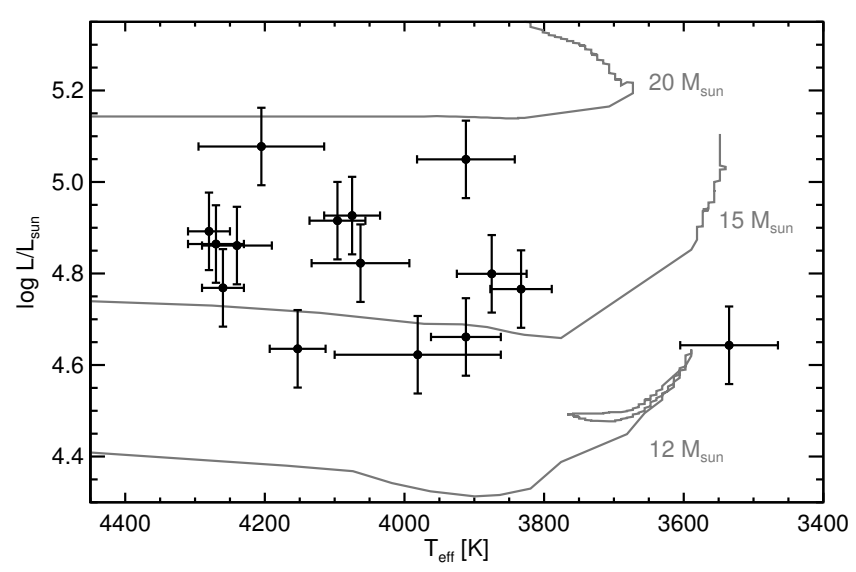

FIG. 8.- Hertzsprung-Russell diagram of program stars for which we have F $814 \mathrm{~W}$ (I) band photometry from Hubble. Temperatures are the parameter fits from our analysis procedure and we calculate luminosities using the bolometric correction recipes for RSGs of Davies et al. (2013).

We then overplot evolutionary tracks with solar metallicity adopting the Geneva database of stellar evolutionary models including the effects of rotation (Meynet \& Maeder 2000). We note that all program stars with HST magnitudes fall well within the ranges in temperature and luminosity which are appropriate for RSGs. We calculate stellar masses by interpolating fitted temperatures and gravities and calculated luminosities to the Geneva evolutionary models. Errors in mass are calculated with a monte carlo simulation in which, for 1000 trials, we add noise to $T_{\text {eff }}, \log g$, and luminosity on the scale of our fit uncertainties. The mass values and errors presented in Table 5 represent the median and $1 \sigma$ standard deviation of those monte carlo experiments. This experiment indicates an initial mass range between 12 and $20 \mathrm{M}_{\odot}$.

\section{CONCLUSIONS AND FUTURE WORK}

The quantitative spectroscopy of individual stars in distant galaxies as a way to constrain the evolution of galaxies has long been a dream of stellar astronomers. We believe that with the results presented here we have made a major step forward to turn this dream into reality. The stunning agreement between two completely independent stellar spectroscopic methods analyzing massive stars in different evolutionary phases with different sets of model atmospheres, spectral ranges, ionization stages and spectral lines clearly indicates that extragalactic stellar spectroscopy has matured to become an accurate and powerful tool. With new multi-object NIR spectrographs such as KMOS at the VLT and MOSFIRE at Keck and the "classical" MOS instruments at visual light for BSGs such as FORS/VLT and LRIS/Keck we have now two reliable tools to investigate the chemical evolution of galaxies out to $\sim 7 \mathrm{Mpc}$. With future telescopes with 30 to $40 \mathrm{~m}$ apertures as the TMT or E-ELT and adaptive optics (AO) supported NIR multi-object spectrographs the RSG J-band method can be pushed much further. Evans et al. (2011) have demonstrated that for AO supported KMOS-like spectrographs magnitudes down to J $\sim 24$ mag could be reached with sufficient signal-to-noise in two nights of observing. This will allow studies like the one presented here to be carried out at $50 \mathrm{Mpc}$ distance and will make a significant volume of the local universe accessible for accurate quantitative investigations of the chemical evolution of galaxies.

\section{ACKNOWLEDGMENTS}

JZG and RPK acknowledge support by the National Science Foundation under grant AST-1108906 and the hospitality of the Munich University Observatory where part of this work was carried out. BD is supported by a fellowship from the Royal Astronomical Society. BP is supported in part by the Programme National de Physique Stellaire of the INSU CNRS. SJW and AZB acknowledge funding by the European Union (European Social Fund) and National Resources under the ARISTEIA action of the Operational Programme Education and Lifelong Learning in Greece.

\section{REFERENCES}

Asplund, M., Grevesse, N., Sauval, A. J., \& Scott, P. 2009, ARA\&A, 47, 481

Bergemann, M., Kudritzki, R.-P., Gazak, Z., Davies, B., \& Plez, B. 2014, ArXiv e-prints

Bergemann, M., Kudritzki, R.-P., Plez, B., Davies, B., Lind, K., \& Gazak, Z. 2012, ApJ, 751, 156

Bergemann, M., Kudritzki, R.-P., Würl, M., Plez, B., Davies, B., \& Gazak, Z. 2013, ApJ, 764, 115

Bonanos, A. Z., et al. 2009, AJ, 138, 1003

Bresolin, F. 2011, ApJ, 730, 129

Bresolin, F., Gieren, W., Kudritzki, R., Pietrzyński, G., Urbaneja, M. A., \& Carraro, G. 2009, ApJ, 700, 309

Bresolin, F., Pietrzyński, G., Urbaneja, M. A., Gieren, W., Kudritzki, R., \& Venn, K. A. 2006, ApJ, 648, 1007
Bresolin, F., Urbaneja, M. A., Gieren, W., Pietrzyński, G., \& Kudritzki, R. 2007, ApJ, 671, 2028

Brott, I., et al. 2011, A\&A, 530, A115

Daflon, S., \& Cunha, K. 2004, The Astrophysical Journal, 617, 1115

Dalcanton, J. J., et al. 2009, ApJS, 183, 67

Davies, B., Kudritzki, R., \& Figer, D. F. 2010, MNRAS, 407, 1203

Davies, B., Kudritzki, R., Gazak, J. Z., Plez, B., Bergemann, M., Evans, C., \& Patrick, L. 2014, ApJ

Davies, B., et al. 2013, ApJ, 767, 3

Deharveng, L., Peña, M., Caplan, J., \& Costero, R. 2000, MNRAS, 311, 329

Ekström, S., et al. 2012, A\&A, 537, A146

Eldridge, J. J., Izzard, R. G., \& Tout, C. A. 2008, MNRAS, 384 1109 
TABLE 3

Comparison of NGC 300 Metallicity and Gradient Literature

\begin{tabular}{lccccc}
\hline \hline \multicolumn{1}{c}{ Study } & \multicolumn{2}{c}{ Central Abundance } & \multicolumn{2}{c}{ Metallicity Gradient } & Notes \\
& dex, metals & $12+\log (\mathrm{O} / \mathrm{H})$ & $\mathrm{R} / \mathrm{R}_{25}$ & $\mathrm{dex} \mathrm{kpc}^{-1}$ & \\
\hline Kudritzki et al. (2008) & $-0.07 \pm 0.09$ & $\ldots$ & $-0.44 \pm 0.06$ & $-0.081 \pm 0.011$ & Blue Supergiants, Metals \\
\hline Bresolin et al. (2009) & $\ldots$ & $8.57 \pm 0.02$ & $-0.41 \pm 0.03$ & $-0.077 \pm 0.006$ & $\begin{array}{l}\text { H il regions, auroral oxygen } \\
\text { This work }\end{array}$ \\
\hline Ris & $-0.03 \pm 0.05$ & & $-0.44 \pm 0.08$ & $-0.083 \pm 0.014$ & Red Supergiants, Metals \\
\hline
\end{tabular}

NoтE. - Three independent measurements of the evolution of chemical abundance across the star forming disk of NGC 300. The agreement between BSGs, RSGs, and auroral H II region methods is excellent.

Evans, C. J., Bresolin, F., Urbaneja, M. A., Pietrzyński, G., Gieren, W., \& Kudritzki, R. 2007, ApJ, 659, 1198

Evans, C. J., et al. 2011, A\&A, 527, A50+

Freudling, W., Romaniello, M., Bramich, D. M., Ballester, P., Forchi, V., García-Dabló, C. E., Moehler, S., \& Neeser, M. J. 2013, A\&A, 559, A96

Gazak, J. Z., Davies, B., Kudritzki, R., Bergemann, M., \& Plez, B. 2014, ApJ, 788, 58

Georgy, C., et al. 2013, A\&A, 558, A103

Gieren, W., Pietrzyński, G., Soszyński, I., Bresolin, F., Kudritzki R.-P., Minniti, D., \& Storm, J. 2005, ApJ, 628, 695

Gustafsson, B., Edvardsson, B., Eriksson, K., Jørgensen, U. G., Nordlund, A., \& Plez, B. 2008, A\&A, 486, 951

Hosek, Jr., M. W., et al. 2014, ApJ, 785, 151

Kewley, L. J., \& Ellison, S. L. 2008, ApJ, 681, 1183

Khan, R., Stanek, K. Z., Prieto, J. L., Kochanek, C. S., Thompson, T. A., \& Beacom, J. F. 2010, ApJ, 715, 1094

Kudritzki, R., Urbaneja, M. A., Bresolin, F., Przybilla, N., Gieren, W., \& Pietrzyński, G. 2008, ApJ, 681, 269

Kudritzki, R.-P., Urbaneja, M. A., Bresolin, F., Hosek, Jr., M. W., \& Przybilla, N. 2014, ApJ, 788, 56

Kudritzki, R.-P., Urbaneja, M. A., Gazak, Z., Bresolin, F., Przybilla, N., Gieren, W., \& Pietrzyński, G. 2012, ApJ, 747, 15

Kudritzki, R.-P., Urbaneja, M. A., Gazak, Z., Macri, L., Hosek, Jr., M. W., Bresolin, F., \& Przybilla, N. 2013, ApJ, 779, L20

Lee, H., Skillman, E. D., \& Venn, K. A. 2006, ApJ, 642, 813

Magic, Z., Weiss, A., \& Asplund, M. 2015, A\&A, 573, A89

Mennickent, R. E., Pietrzyński, G., \& Gieren, W. 2004, MNRAS, 350,679

Meynet, G., \& Maeder, A. 2000, A\&A, 361, 101

-. 2002, A\&A, 390, 561
Noll, S., Kausch, W., Kimeswenger, S., Barden, M., Jones, A. M., Modigliani, A., Szyszka, C., \& Taylor, J. 2014, A\&A, 567, A25

Patrick, L., Evans, C., Davies, B., Kudritzki, R., Gazak, J. Z., Bergemann, M., Plez, B., \& Ferguson, A. 2014, ApJ, in press

Pietrzyński, G., Gieren, W., Fouqué, P., \& Pont, F. 2001, A\&A, 371,497

Rolleston, W. R. J., Smartt, S. J., Dufton, P. L., \& Ryans, R. S. I. 2000, A\&A, 363, 537

Russeil, D. 2003, A\&A, 397, 133

Skrutskie, M. F., et al. 2006, AJ, 131, 1163

Stasińska, G. 2005, A\&A, 434, 507

Trampedach, R., Stein, R. F., Christensen-Dalsgaard, J., Nordlund, Å., \& Asplund, M. 2014, MNRAS, 445, 4366

U, V., Urbaneja, M. A., Kudritzki, R., Jacobs, B. A., Bresolin, F., \& Przybilla, N. 2009, ApJ, 704, 1120

Urbaneja, M. A., Herrero, A., Kudritzki, R.-P., Najarro, F., Smartt, S. J., Puls, J., Lennon, D. J., \& Corral, L. J. 2005, The Astrophysical Journal, 635, 311

Urbaneja, M. A., Kudritzki, R., Bresolin, F., Przybilla, N., Gieren, W., \& Pietrzyński, G. 2008, ApJ, 684, 118

Verhoelst, T., van der Zypen, N., Hony, S., Decin, L., Cami, J., \& Eriksson, K. 2009, A\&A, 498, 127

Vilchez, J. M., Pagel, B. E. J., Diaz, A. I., Terlevich, E., \& Edmunds, M. G. 1988, MNRAS, 235, 633

Wegner, M., \& Muschielok, B. 2008, in Society of Photo-Optical Instrumentation Engineers (SPIE) Conference Series, Vol. 7019, Society of Photo-Optical Instrumentation Engineers (SPIE) Conference Series, 0

Westmeier, T., Braun, R., \& Koribalski, B. S. 2011, MNRAS, 410,2217

Zurita, A., \& Bresolin, F. 2012, MNRAS, 427, 1463 
TABLE 4

\begin{tabular}{|c|c|c|c|c|c|c|c|}
\hline Target & Alt. Designation & $\begin{array}{c}\text { Right Ascension } \\
\text { HH:MM:SS }\end{array}$ & $\begin{array}{l}\text { Declination } \\
\text { DD:MM:SS }\end{array}$ & $\mathrm{R} / \mathrm{R}_{25}$ & $\begin{array}{c}\mathrm{HST} \text { F814W } \\
\text { mag }\end{array}$ & $\begin{array}{c}\text { IRAC Band } 1 \\
\text { mag }\end{array}$ & $\begin{array}{c}\text { IRAC Band } 2 \\
\text { mag }\end{array}$ \\
\hline 010 & $\cdots$ & $00: 54: 52.07$ & $-37: 40: 32.1$ & 0.07 & $18.220 \pm 0.001$ & $14.61 \pm 0.03$ & $14.61 \pm 0.05$ \\
\hline 022 & $\cdots$ & $00: 54: 53.83$ & $-37: 40: 08.6$ & 0.12 & $18.956 \pm 0.002$ & $15.88 \pm 0.03$ & $16.03 \pm 0.05$ \\
\hline 024 & $\cdots$ & $00: 54: 53.12$ & $-37: 39: 28.2$ & 0.20 & $18.977 \pm 0.002$ & $15.78 \pm 0.02$ & $15.47 \pm 0.07$ \\
\hline 007 & J00544792-3739105a & $00: 54: 47.90$ & $-37: 39: 10.0$ & 0.25 & $17.858 \pm 0.001$ & $15.35 \pm 0.02$ & $15.45 \pm 0.05$ \\
\hline 013 & $\ldots$ & $00: 54: 49.58$ & $-37: 38: 50.5$ & 0.28 & $18.534 \pm 0.001$ & $\ldots$ & $\ldots$ \\
\hline 127 & J00544267-3739172a & $00: 54: 42.64$ & $-37: 39: 16.9$ & 0.29 & $\ldots$ & $14.71 \pm 0.02$ & $14.76 \pm 0.04$ \\
\hline 011 & $\ldots$ & $00: 54: 49.91$ & $-37: 38: 27.3$ & 0.33 & $18.471 \pm 0.001$ & $15.90 \pm 0.02$ & $15.84 \pm 0.04$ \\
\hline 009 & MPG2004 $72^{\mathrm{b}}$ & $00: 54: 45.40$ & $-37: 38: 35.2$ & 0.33 & $18.190 \pm 0.001$ & $15.66 \pm 0.04$ & $15.70 \pm 0.05$ \\
\hline 014 & $\ldots$ & $00: 54: 48.56$ & $-37: 38: 02.0$ & 0.38 & $18.617 \pm 0.001$ & $15.46 \pm 0.02$ & $15.29 \pm 0.06$ \\
\hline 006 & $\cdots$ & $00: 54: 47.96$ & $-37: 37: 39.2$ & 0.43 & $17.782 \pm 0.001$ & $15.67 \pm 0.02$ & $15.72 \pm 0.04$ \\
\hline 101 & $\cdots$ & $00: 55: 02.12$ & $-37: 37: 50.3$ & 0.49 & $\ldots$ & $15.28 \pm 0.02$ & $15.30 \pm 0.05$ \\
\hline 102 & $\cdots$ & $00: 54: 53.72$ & $-37: 36: 47.5$ & 0.55 & $\cdots$ & $15.22 \pm 0.02$ & $15.30 \pm 0.04$ \\
\hline 133 & J00543092-3737570a & $00: 54: 30.96$ & $-37: 37: 57.0$ & 0.56 & $\cdots$ & $15.57 \pm 0.02$ & $15.58 \pm 0.05$ \\
\hline 130 & J00542809-3738431 ${ }^{\mathrm{a}}$ & $00: 54: 28.10$ & $-37: 38: 42.8$ & 0.57 & $\cdots$ & $\cdots$ & $\cdots$ \\
\hline 126 & $\ldots$ & $00: 54: 25.54$ & $-37: 39: 54.6$ & 0.59 & $\cdots$ & $15.22 \pm 0.02$ & $15.26 \pm 0.02$ \\
\hline 135 & $\ldots$ & $00: 54: 29.38$ & $-37: 37: 55.9$ & 0.59 & $\cdots$ & $15.26 \pm 0.02$ & $15.35 \pm 0.06$ \\
\hline 134 & MPG2004 31 & $00: 54: 28.25$ & $-37: 37: 54.3$ & 0.61 & $\ldots$ & $\ldots$ & $\ldots$ \\
\hline 039 & $\ldots$ & $00: 54: 25.76$ & $-37: 37: 58.7$ & 0.65 & $18.367 \pm 0.003$ & $15.99 \pm 0.02$ & $15.83 \pm 0.07$ \\
\hline 128 & $\cdots$ & $00: 54: 22.00$ & $-37: 39: 08.0$ & 0.67 & $\ldots$ & $14.91 \pm 0.04$ & $14.87 \pm 0.05$ \\
\hline 129 & J00541708-3738551 & $00: 54: 17.06$ & $-37: 38: 54.6$ & 0.78 & $\ldots$ & $\ldots$ & $\ldots$ \\
\hline 139 & $\cdots$ & $00: 54: 26.77$ & $-37: 35: 04.3$ & 0.87 & $18.283 \pm 0.001$ & $\cdots$ & $\cdots$ \\
\hline 034 & MPG2004 15 & $00: 54: 18.59$ & $-37: 35: 38.4$ & 0.92 & $18.624 \pm 0.001$ & $\cdots$ & $\cdots$ \\
\hline 055 & $\ldots$ & $00: 54: 22.70$ & $-37: 34: 40.0$ & 0.95 & $19.012 \pm 0.001$ & $\cdots$ & $\cdots$ \\
\hline 132 & $\cdots$ & 00:54:08.74 & $-37: 38: 02.9$ & 0.96 & $\ldots$ & $\cdots$ & $\cdots$ \\
\hline 140 & $\mathrm{~J} 00541835-3735024^{\mathrm{a}}$ & $00: 54: 18.34$ & $-37: 35: 02.0$ & 0.97 & $\ldots$ & $\cdots$ & $\cdots$ \\
\hline 031 & $\ldots$ & $00: 54: 23.27$ & $-37: 34: 20.3$ & 0.97 & $18.358 \pm 0.001$ & $\cdots$ & $\cdots$ \\
\hline 037 & $\cdots$ & $00: 54: 16.18$ & $-37: 34: 51.6$ & 1.01 & $18.907 \pm 0.002$ & $\cdots$ & $\cdots$ \\
\hline
\end{tabular}

Note. - For objects with no HST F814W entry HST photometry was either not available or uncertain.

a Skrutskie et al. (2006)

b Mennickent et al. (2004)

TABLE 5

\begin{tabular}{|c|c|c|}
\hline Target & $\begin{array}{l}\text { Mass } \\
\mathrm{M}_{\odot}\end{array}$ & $\begin{array}{l}\text { Luminosity } \\
\log \mathrm{L} / \mathrm{L}_{\odot}\end{array}$ \\
\hline 010 & $17.4 \pm 1.0$ & $4.92 \pm 0.08$ \\
\hline 022 & $12.3 \pm 0.8$ & $4.64 \pm 0.08$ \\
\hline 024 & $14.3 \pm 0.8$ & $4.64 \pm 0.08$ \\
\hline 007 & $18.8 \pm 1.1$ & $5.05 \pm 0.08$ \\
\hline 013 & $16.1 \pm 0.9$ & $4.80 \pm 0.08$ \\
\hline 127 & $\ldots$ & \\
\hline 011 & $16.3 \pm 0.9$ & $4.82 \pm 0.08$ \\
\hline 009 & $17.5 \pm 1.0$ & $4.93 \pm 0.08$ \\
\hline 014 & $15.5 \pm 0.9$ & $4.77 \pm 0.08$ \\
\hline 006 & $19.2 \pm 1.2$ & $5.08 \pm 0.08$ \\
\hline 101 & $\ldots$ & $\ldots$ \\
\hline 102 & $\ldots$ & $\ldots$ \\
\hline 133 & $\ldots$ & $\ldots$ \\
\hline 130 & $\ldots$ & $\ldots$ \\
\hline 126 & $\ldots$ & $\ldots$ \\
\hline 135 & $\ldots$ & $\cdots$ \\
\hline 134 & $\ldots$ & $\ldots$ \\
\hline 039 & $16.6 \pm 0.9$ & $4.86 \pm 0.08$ \\
\hline 128 & $\ldots$ & $\ldots$ \\
\hline 129 & $\ldots$ & $\ldots$ \\
\hline 139 & $16.9 \pm 1.0$ & $4.89 \pm 0.08$ \\
\hline 034 & $15.8 \pm 0.8$ & $4.77 \pm 0.08$ \\
\hline 055 & $14.4 \pm 0.8$ & $4.62 \pm 0.08$ \\
\hline 132 & $\ldots$ & $\ldots$ \\
\hline 140 & $\ldots$ & $\ldots$ \\
\hline 031 & $16.7 \pm 1.0$ & $4.86 \pm 0.08$ \\
\hline 037 & $14.8 \pm 0.8$ & $4.66 \pm 0.08$ \\
\hline
\end{tabular}

Note. - 